\title{
Daytime polysomnogram diagnosis of sleep disorders
}

\author{
G BROWNE GOODE, HOWARD M SLYTER \\ From the Department of Neurology, Kaiser-Permanente Medical Center, San Francisco, California, USA
}

SUMMARY The daytime polysomnogram was used to evaluate 310 consecutive patients with suspected sleep disorders, referred mainly because of excessive daytime sleepiness. Abnormalities consistent with pathologic sleep apnoea were present in 102 cases, and with narcolepsy-cataplexy in 49 cases. The daytime polysomnogram is a readily accessible, accurate, and cost-effective method for diagnosing many sleep disorders.

The traditional methods for diagnosing sleep disorders are all-night and 24 hour polygraph recordings. These methods require many personnel, considerable space, and a great deal of expense. Often inconvenient to the patient, they can be performed only in a few diagnostic centres and are poorly accessible to most.

In search of more effective ways to diagnose sleep disorders, we studied the utility of daytime nap polygraph recordings in the electroencephalography (EEG) laboratory of a general hospital. A preliminary report of our findings has been published. ${ }^{1} \mathrm{We}$ now report the results of using the daytime polysomnogram in 310 consecutive patients with suspected sleep disorders. This simplified method is effective in the diagnosis of sleep apnoea and narcolepsycataplexy.

\section{Methods}

The clinical diagnosis of definite sleep apnoea required daytime hypersomnolence, loud snoring and observed apnoea. Possible sleep apnoea was diagnosed in hypersomnolent male patients who had a family history of sleep apnoea, were overweight, or awakened frequently at night. The clinical diagnosis of definite narcolepsy required the presence of daytime hypersomnolence and cataplexy. Possible narcolepsy was diagnosed if a patient with hypersomnolence had frequent sleep paralysis or a family history of definite narcolepsy.

We collected 310 cases during the 30 -month period ending in February, 1982. Most patients were Kaiser Foundation Health Plan members in Northern California referred by their local neurologist or internist at one of our

Address for reprint requests: Dr GB Goode, Department of Neurology, Kaiser-Permanente Medical Center, 2200 O'Farrell Street, San Francisco, CA 94115, USA.

Received 18 May 1982 and in revised form 28 September 1982. Accepted 18 October 1982. fourteen facilities; a few were private patients in the community.

On the day of the test, the patient arose early and refrained from naps. Most patients retired at the usual time the night before and awoke at 6 am on the day of the test. The quality and duration of the prior night's sleep and the number of awakenings was recorded by the technician. A 90 -minute postprandial test with recording time of around 60 minutes was scheduled in the early afternoon. A minimum of 30 minutes sleep was attempted. The test was considered unsatisfactory if the patient slept less than 20 minutes or if the sleep was frequently interrupted. Prior to the test, less than $20 \%$ of patients were receiving medication. Any stimulant medication was discontinued before the test, in most cases for more than seven days. Other medications, such as tricyclic drugs, were discontinued one to two weeks before the test.

The polygraphs were performed with a 16-channel clinical EEG instrument. One channel each was used to monitor an electrocardiogram (ECG), an anterior neck muscle electromyogram (EMG), right and left ocular movements, thoracic respirations, right and left nostril airflow, and mouth air flow. Mouth and nostril air flow determinations were made by thermistors, and thoracic movements were monitored by a bellows-type strain-gauge instrument. Six channels were used for scalp EEG recordings. Oxygen saturation was recorded in many cases by use of an ear oximeter.

We encountered almost no technical problems after a few months of experience. The sleep recorded was mainly stage I and stage II; there was some rapid eye movement (REM) sleep and rare stage III sleep. If the patient slept insufficiently or if the test was normal but a sleep disorder was strongly suspected, we repeated the test, sometimes with sleep deprivation.

The polysomnogram diagnosis of pathologic sleep apnoea required that apnoea had to occur at other times than during sleep onset (the first 10 minutes of sleep) and REM sleep. Central apnoea had to occur more than 15 times in men and more than five times in women. Obstructive and mixed types of apnoea had to occur more than twice in both sexes. These criteria were based on our experience with control and known pathological cases as 
well as on a review of the literature of daytime polysomnograms in asymptomatic subjects. The polysomnogram diagnosis of narcolepsy-cataplexy required at least one episode of unequivocal sleep-onset REM activity. Hypopnoea was defined as respiratory excursions less than one third the amplitude of the baseline excursions.

A modified Multiple Sleep Latency Test ${ }^{2}$ was sometimes performed in narcolepsy-cataplexy suspects. After 20 minutes of sleep, the patient was repeatedly awakened every 10 minutes for a minute or more then allowed to return to sleep.

\section{Results}

The primary reason for referral was excessive daytime sleepiness in $90 \%$, insomnia in $3 \%$, observed or symptomatic apnoea in $3 \%$, cataplexy and/ or sleep paralysis in $2 \%$, and miscellaneous reasons in $2 \%$. Excessive daytime sleepiness was the primary reason for referral in $90 \%$ of the sleep apnoea cases, $100 \%$ of the narcolepsy-cataplexy cases, and $85 \%$ of the cases with normal studies. Although the daytime sleepiness tended to be more severe and precipitous in narcolepsy-cataplexy cases, no features of the sleepiness were specific enough to be clinically useful in distinguishing between sleep apnoea and narcolepsy-cataplexy.

The test results in the 310 patients are shown in table 1. Of sleep apnoea cases diagnosed by polysomnography, $79 \%$ were correctly diagnosed clinically before the test. The accurate clinical diagnosis of sleep apnoea often depended on a firsthand description of the patient's sleep. Of the narcolepsy cases diagnosed by polysomnography, $51 \%$ were also diagnosed clinically. If cataplexy was subtle or was not present, narcolepsy was often misdiagnosed. Repeated nap polygraphs increased the diagnostic yield by $20 \%$ for narcolepsy-cataplexy but only by $2 \%$ for sleep apnoea.

Sleep apnoea Pathological sleep apnoea was diagnosed in 102 patients. There were 13 additional cases of possible sleep apnoea. These patients did not qualify as definite because they had insufficient sleep recorded and/or an insufficient number of apnoeic

Table 1 Polysomnogram diagnosis in 310 patients

\begin{tabular}{lcc}
\hline Diagnosed & $\begin{array}{l}\text { Based on } \\
\text { initial clinical } \\
\text { evaluation }\end{array}$ & $\begin{array}{l}\text { Based on } \\
\text { all polysomnograms }\end{array}$ \\
\hline Sleep apnoea* & 82 & 102 \\
Narcolepsy-cataplexy* & 25 & 49 \\
Cheyne-Stokes & 1 & 2 \\
Epileptiform & 0 & 1 \\
No diagnosis & 202 & 156 \\
\hline
\end{tabular}

* 15 cases had polysomnogram evidence of both sleep apnoea and narcolepsy-cataplexy; none were clinically diagnosed as having both conditions. episodes. The sleep apnoea episodes lasted from the conventional minimum duration of 10 seconds to as long as 140 seconds. Most apnoea episodes lasted from 15 to 30 seconds. The percentage of total sleep time spent in apnoea ranged from less than $10 \%$ to over $80 \%$ and in most cases was between $30 \%$ and $60 \%$. In many cases, the apnoea episodes were periodic during part of the test.

The records in many cases of sleep apnoea contained episodes of hypopnoea, and hypopnoea alone occurred in a few cases without apnoea and was not regarded as abnormal in this study. None of our hypopnoea (without apnoea) patients studied with an ear oximeter had significant decrease in oxygen saturation. In many patients with brief apnoea, the oxygen saturation decreased from around $95 \%$ to $80 \%$; with apnoea longer than 30 seconds, the reduction was sometimes as low as $60 \%$.

Table 2 shows the types of sleep apnoea diagnosed in the 102 cases. Many patients had a mixture of two or three types of apnoea during one polysomnogram, although one type of apnoea usually predominated. On repeated testing, a change from predominantly one type of apnoea to another was present in several cases. In untreated sleep apnoea patients, satisfactory serial records were consistently abnormal, unlike those of the narcolepsy-cataplexy patients in $\frac{\stackrel{\circ}{\circ}}{\sigma}$ whom the abnormalities were present inconsistently. Several patients with pathological sleep apnoea in the daytime naps were studied elsewhere by all-night $\delta$ recordings, and the abnormalities were confirmed.

Of the 102 patients with sleep apnoea, 83 were male and 19 female. Ages ranged from 20 to 72 years, $\stackrel{\rightleftharpoons}{\rightleftharpoons}$ and the median age was 48 years. Many, but not all, of the 102 patients were overweight: 28 weighed 200 250 pounds, 20 weighed $250-300$ pounds, and six weighed more than 300 pounds. In three patients, the diagnosis fulfilled criteria for the cardiopulmonaryobesity (Pickwickian) syndrome. Fifteen patients had a thin body habitus.

Narcolepsy-cataplexy Polysomnogram evidence of narcolepsy-cataplexy was present in 49 patients: 37 male and 12 female. Their ages ranged from 14 to 74 years. Of the 49 cases, fifteen had pathologic sleep apnoea in addition to sleep-onset REM activity. The REM episodes consisted of multiple, rapid conjugate eye movements, and most episodes were accompanied by myoclonus and attenuated or absent EMG

Table 2 Types of sleep apnoea in 102 patients

\begin{tabular}{llcl}
\hline Apnoea type & Total & Only & Primary \\
\hline Central & 74 & 29 & 54 \\
Obstructive & 56 & 13 & 42 \\
Mixed & 33 & 0 & 6 \\
\hline
\end{tabular}


activity. Brief central apnoeic episodes occurred during many REM episodes. The yield of abnormalities increased when patients were advised to discontinue analeptic medications before the test and to take no naps on the day of the test.

The REM activity occurred at only some of the sleep onsets. The more sleep onsets recorded, the higher the yield of sleep-onset REM. Many patients spontaneously awakened during the test and then slept again. Sleep-onset REM sometimes followed the spontaneous or induced awakenings.

The 15 patients with pathological sleep apnoea who also had sleep onset REM activity demonstrated some clinical features as well that suggest an overlap between the two disorders. These cases are the subject of a separate communication.

The sleep latency, which is the time from beginning of the recording to the first EEG evidence of sustained sleep, was measured in most cases. If the sleep latency was more than 10 minutes, the polysomnogram was nearly always normal and often little sleep occurred. Sleep latencies of less than 10 minutes did not distinguish among patients with sleep apnoea, narcolepsy-cataplexy, and normal tests.

Cheyne-Stokes respiration were diagnosed in two patients with metabolic encephalopathy and lethargy. The apnoea episodes resembled those seen in central sleep apnoea but were distinguished by strict periodicity and gradual increasing respiratory excursions at each resumption of breathing and by gradual tapering of the respiratory excursions at the end of each respiratory period.

One patient with suspected sleep disorder (because of brief lapses of awareness) was found to have a normal polysomnogram except for generalised spike and wave discharges. Two of the patients with sleep apnoea had a history of generalised seizures, but no epileptiform abnormalities were present in their polygraphs.

Of the 310 patients who had daytime polysomnograms, 47 had repeated testing: 41 had two tests, five had three tests, and one had more than three. A total of 364 tests were performed among the 310 patients. Of the 174 initial polysomnograms considered normal, 77 were considered satisfactory and 97 were considered unsatisfactory. Most unsatisfactory studies were the result of the patient's insufficient sleep. Of the 97 cases with initially unsatisfactory records, 30 were repeated; 15 were satisfactory but 15 remained unsatisfactory. Seven initially satisfactory studies were repeated, and six remained satisfactory. Ninety-two of the 174 normal studies had at least one satisfactory record. Three patients with normal recordings were studied elsewhere by all-night or 24hour studies and were found to have sleep-onset REM.

\section{Discussion}

In this series of symptomatic patients studied by daytime polysomnograms, sleep apnoea was considerably more prevalent than narcolepsy-cataplexy. Our findings confirm those of a very large multicentre study, which found sleep apnoea to be considerably more prevalent than narcolepsycataplexy. ${ }^{3}$ If detailed information was available, including a firsthand description of the patient's sleep, most cases of sleep apnoea and narcolepsycataplexy could be distinguished with reasonable certainty on clinical grounds. Many other cases could be distinguished by the polysomnogram findings only. These cases demonstrated a large proportion of central apnoea as the primary or only type. This finding is probably a reflection of diurnal rather than nocturnal testing and has been reported by others. ${ }^{4}$

This study demonstrates the successful utilisation of the daytime polysomnogram for diagnosing many sleep disorders. Using a few inexpensive monitoring devices and a short period of laboratory time, many general hospital EEG laboratories can effectively diagnose sleep disorders. Three or four daytime studies can be performed at less than the cost of one all-night recording. Thus, repeated testing, if necessary, is cost-effective.

Long-term follow-up of many of our cases is incomplete, and there is concern that some patients with unsatisfactory studies could have a sleep disorder. However, data on cases evaluated by the authors indicate that few patients with unsatisfactory tests because of insufficient sleep have sleep apnoea or narcolepsy.

Daytime nap recordings appear to detect sleep apnoea and many cases of narcolepsy reliably, but validation of the reliability of this technique awaits evaluation of a group of patients studied by both nap and all-night recordings. Our experience indicates that daytime sleep recordings may be the only study necessary to diagnose most sleep disorder cases. At least as a screening test, the daytime polysomnogram has potential for widespread application.

\section{References}

${ }^{1}$ Goode G, Slyter H. Diagnosis of sleep apnea by daytime polysomnogram. Ann Neurol 1980;8:118.

${ }^{2}$ Richardson GS, Karskadon MA, Slagg W, van den Hoed J, Dement WC, Mitler MM. Excessive daytime sleepiness in man: Multiple sleep latency measurement in narcoleptic and control subjects. Electroencephalogr Clin Neurophysiol 1978;45:621-7.

${ }^{3}$ Coleman RM, Roffwarg HP, Kennedy SJ, et al. Sleepwake disorders based on a ploysomnographic diagnosis. JAMA 1982;247:997-1003.

${ }^{4}$ Chokroverty S, Sharp JT. Primary sleep apnoea syndrome. J Neurol Neurosurg Psychiatry 1981;44:970-82. 